\title{
Effects of Perineural Capsaicin Treatment on Cardiopulmonary Reflexes Elicited by Laryngeal Instillations of Capsaicin and Distilled Water in Sevoflurane-Anesthetized Dogs
}

\author{
Tatsushi MUTOH, Arata KANAMARU ${ }^{1)}$, Kentaro KOJIMA, Ryohei NISHIMURA, Nobuo SASAKI and Hirokazu \\ $\mathrm{TSUBONE}^{1)} *$ \\ Departments of Veterinary Surgery and ${ }^{1)}$ Comparative Pathophysiology, Graduate School of Agricultural and Life Sciences, The \\ University of Tokyo, Bunkyo-ku, Tokyo 113-8657, Japan
}

(Received 16 November 1999/Accepted 18 February 2000)

ABSTRACT. The aim of this study was to determine the effect of perineural capsaicin (CAPS) treatment on cardiopulmonary reflexes elicited by topical laryngeal instillation of CAPS and distilled water (DW) in sevoflurane-anesthetized dogs. Cardiopulmonary reflexes elicited by CAPS $(10 \mu \mathrm{g} / \mathrm{m} l, 10 \mathrm{~m} l)$ were attenuated by perineural CAPS treatment to the superior laryngeal nerves (SLNs) $(P<0.05)$, whereas those by DW $(10 \mathrm{~m} l)$ remained unaffected $(P>0.05)$. The reflex responses to DW that remained even after the perineural CAPS treatment were eliminated by laryngeal anesthesia with lidocaine. These results suggest that cardiopulmonary reflexes from the laryngeal mucosa elicited by CAPS instillation can be blocked by perineural CAPS treatment to the SLNs, which may result from inhibition of the laryngeal CAPS-sensitive C-fiber afferents.-KEY wORDS: canine, control of breathing, laryngeal afferents.

The larynx is a potent reflexogenic region of the upper airway that is rich in sensory afferents and elicits various airway defence reflexes such as apnea, coughing, glottal closure, mucus hypersecretion, bronchoconstriction, bradycardia and hypertension [10]. Among several types of mechanoreceptors described in the larynx, capsaicin (CAPS)-sensitive $\mathrm{C}$-fibers and water responsive rapidly adapting 'irritant' receptors are thought to have primary role in eliciting these airway reflexes. Whereas there are several studies which suggest the behavior of the CAPS-sensitive C-fibers and water-responsive receptors electrophysiologically $[4,10]$, the reflex data to support their contribution is still lacking.

In a recent report, we have demonstrated that perineural CAPS treatment to the SLNs can block C-wave afferent compound action potentials in anesthetized dogs [6]. We hypothesized that if the afferent information via the Cfibers were preferably blocked by the perineural CAPS treatment, then the resultant cardiopulmonary reflexes would be attenuated or extinguished without affecting other myelinated fiber components such as water-responsive receptors. Thus the purpose of this study was to determine the effect of perineural CAPS treatment on cardiopulmonary reflexes elicited by topical laryngeal instillation of CAPS and distilled water (DW) in dogs.

Animals and anesthesia: Six healthy beagles (3 females and 3 males) were studied. Their mean age was 13.5 (range, 10 to 15 ) months and mean body weight was 8.6

\footnotetext{
* Correspondence to: Tsubone, H., Department of Comparative Pathophysiology, Graduate School of Agricultural and Life Sciences, The University of Tokyo, 1-1-1 Yayoi, Bunkyo-ku, Tokyo 113-8657, Japan.
}

(range, 6.5 to 10.0 ) $\mathrm{kg}$. Food was withheld for $12 \mathrm{hr}$ before experiments. All the dogs were subjected to permanent tracheostomy 2-3 weeks before experiments according to the method described previously [3, 5]. Dogs were premedicated and anesthetized according to the method previously described [6].

General setup: Laryngeal mask (10 mm ID; LM1003, The Laryngeal Mask Co, UK) was introduced to cover the larynx including the epiglottis. A cuffed tracheostomy tube (7.0-8.0 $\mathrm{mm}$ ID) was introduced into the upper trachea through a tracheostoma. The tip of the upper tracheostomy tube was carefully positioned at the level of cricoid cartilage in order to isolate the larynx functionally. Respiratory airflow was measured with the differential pressure transducer (DD 102A, Toyoda Machine Works, Japan) through two sidearms connected to the tracheostomy tube, and integrated with an A-D converter (Mac Lab Scope, BRC Inc, Japan) connected to the Macintosh computer (PowerBook 5300cs, Apple Computer Inc, USA) to give tidal volume $\left(\mathrm{V}_{\mathrm{T}}\right)$. Intratracheal pressure was measured with a pressure transducer (DD102A, Toyoda Machine Works, Tokyo, Japan) through a sidearm attached to the cuffed tracheal tube. Expired ventilation $\left(\dot{\mathrm{V}}_{\mathrm{E}}\right)$ was calculated from the value of $\mathrm{V}_{\mathrm{T}}$ and total cycle duration. A saline-filled polyethylene catheter ( $2 \mathrm{~mm}$ ID) was placed in the middle portion of the esophagus and connected to a pressure transducer (DX-300, Nihon Kohden, Japan) to record esophageal pressure. Inspiration time $\left(\mathrm{T}_{\mathrm{I}}\right)$ and expiration time $\left(\mathrm{T}_{\mathrm{E}}\right)$ were measured from the tracing of esophageal pressure as the end of one inspiration to the beginning of the next inspiration. Arterial blood pressure was monitored by a pressure transducer (DX-300, Nihon 
Kohden, Japan), connected to a 20-G catheter inserted into the femoral artery. Mean arterial blood pressure (MAP) was calculated every $5 \mathrm{sec}$ of bin as the sum of diastolic pressure and one-third of pulse pressure. Heart rate (HR) was derived electrophysiologically from the blood pressure signal. All the signals were displayed on a thermal-array recorder (RT 3100N, NEC san-ei, Japan) and recorded by a magnetic tape recorder (PC 208, SONY, Japan). Tidal $\mathrm{PcO}_{2}$ was sampled through the same line as that use to measure the anesthetic concentration, and end-tidal $\mathrm{PcO}_{2}\left(\mathrm{PETCO}_{2}\right)$ was monitored by use of the infrared gas analyzer (AGM103 Capnomac, Datex, Finland). During the experiment, rectal temperature was maintained at $37 \pm 1^{\circ} \mathrm{C}$, using a warming blanket. Lactated Ringer's solution was infused at a rate of $10 \mathrm{~m} / / \mathrm{kg} / \mathrm{hr}$ into the cephalic vein through the needle-catheter.

Perineural CAPS treatment: Bilateral internal branches of the SLN were exposed and desheathed as described previously [6]. Cotton pledgets soaked in a digestive solution consisting of Krebs solution containing collagenase and hyaluronidase were placed on bilateral SLNs for $20 \mathrm{~min}$ to remove connective tissue and to increase segment permeability. Then the digestive solution was removed by washing with saline and cotton pledgets containing a $100 \mu \mathrm{g} / \mathrm{m} l$ CAPS solution in a vehicle of $10 \%$ ethanol, $10 \%$ Tween 80 , and $80 \%$ saline were placed on the nerve segments in order to block the C-fiber afferents [6].

Experimental protocol: At the commencement of each trial, $\mathrm{PETCO}_{2}$ was adjusted between $35-40 \mathrm{mmHg}$ by assisted manual ventilation. After a control period of more than one minute, a $10 \mathrm{~m} l$ of CAPS solution $(10 \mu \mathrm{g} / \mathrm{ml}$, a diluted solution of $100 \mu \mathrm{g} / \mathrm{m} l$ CAPS) was instilled into the isolated larynx with a catheter with multiple holes at the distal port inserted through the laryngeal mask. Then the same volume of distilled water (DW) was instilled in the same manner approximately $15 \mathrm{~min}$ after the CAPS instillation. This procedure was performed 3 times at an interval of $20 \mathrm{~min}$ or more: 1) before perineural CAPS treatment (non-treatment), 2) after perineural CAPS treatment, 3) after laryngeal anesthesia with lidocaine. The order of each trial, the time interval and the dose of CAPS to minimize the tachyphilaxis were determined by both pilot and previous studies [7, 8]. Isotonic $\mathrm{NaCl}$ solution $(0.9 \%)$ was used for rinsing the laryngeal lumen between trials. For laryngeal anesthesia, $5 \mathrm{ml}$ of $2 \%$ lidocaine was aerosolized with an ultrasonic nebulizer driven by the $\mathrm{O}_{2}(5$ $l / \mathrm{min}$, output $2.5 \mathrm{~m} l / \mathrm{min})$, producing particles approximately $5 \mu \mathrm{m}$ in size, and passed through the isolated larynx for $2 \mathrm{~min}$ before instillation trials. At the end of the experiments, all the dogs were euthanized by an overdose injection of pentobarbital $(50 \mathrm{mg} / \mathrm{kg}$, i.v.).

Data analysis: Respiratory variables $\left(\mathrm{T}_{\mathrm{I}}, \mathrm{T}_{\mathrm{E}}, \mathrm{V}_{\mathrm{T}}, \dot{\mathrm{V}}_{\mathrm{E}}\right)$ were all analyzed on a breath-by-breath basis. Control values for the respiratory variables were averaged over three consecutive breaths immediately before each trial. Control values for cardiovascular values (HR and MAP) were averaged for $60 \mathrm{sec}$ before each trial. Peak responses after the onset of each trial were taken into account. For comparisons of the differences in control values, one-factor ANOVA followed by a Tukey's post hoc test was used. To determine whether the changes from control to the peak responses after each trial were statistically significant, we used a paired Student's $t$-test. The evoked changes from the control values $(\Delta)$ were compared by use of a one-way ANOVA for repeated measures with post hoc (Tukey's) test. Values of $P<0.05$ were considered statistically significant. All data were expressed as means \pm SE, unless otherwise indicated.

There were no significant differences in control values ( $\mathrm{T}_{\mathrm{E}}$ and $\dot{\mathrm{V}}_{\mathrm{E}}: 3$ consecutive breaths before instillation, MAP and HR: $60 \mathrm{sec}$ before instillation) among trials. Figure 1 shows an example of cardiopulmonary reflexes by instillation of CAPS and DW into the larynx with and without pretreatment of perineural CAPS to the SLNs. Laryngeal instillation of CAPS induced an inhibition of breathing characterized by an increase in $T_{E}$ and a resultant decrease in $\dot{\mathrm{V}}_{\mathrm{E}}$; and a decrease in $\mathrm{HR}$ and an increase in MAP for cardiovascular reflexes (Fig. 2, non-treatment). No significant changes in $T_{I}$ or $V_{T}$ were observed by instillation of either CAPS or DW (data not shown). Cardiopulmonary reflexes elicited by the CAPS instillation were significantly attenuated after perineural CAPS treatment $(P<0.05$ vs non-treatment), whereas those to DW remained unaffected ( $P>0.05$ vs non-treatment) (Fig. 2). All the reflex responses to DW instillation that remained even after perineural CAPS treatment were blocked by laryngeal anesthesia with lidocaine $(P<0.01$ vs nontreatment) (Fig. 2).

Airway protective reflexes characterized by a prolongation of $\mathrm{T}_{\mathrm{E}}$ (apnea) and the resultant ventilatory depression are common findings in previous reflex studies which applied CAPS and DW into the larynx of anesthetized rats and $\operatorname{dogs}[1,3,10]$. Cardiovascular reflexes (hypertension and bradycardia) also appear to be a characteristic response from the larynx to CAPS and DW instillations caused by sympathetic nervous system activation and associated baroreflex response [1,3].

The elicitation of similar reflex cardiopulmonary responses to CAPS and DW suggests that the central processing associated with afferent stimuli may ultimately project to a common efferent pathway to exhibit the reflexes via the somatic and autonomic nervous systems. The afferents from the vagal pathway generally transmit impulses to the nucleus tractus solitarius (NTS), and this electrical activity influences the medullary respiratory neuron groups and dorsal nucleus of the vagus to elicit various reflex responses [9].

It is well known that afferent innervation of the larynx is primarily supplied by the internal branch of the SLNs [10]. Our results suggest that significant reduction of reflex cardiopulmonary responses to CAPS with less responsiveness to DW caused by perineural CAPS treatment to the SLNs indicates a preferable blocking effect of the CAPS-sensitive afferents, because CAPS is widely 


\section{Before perineural CAPS}
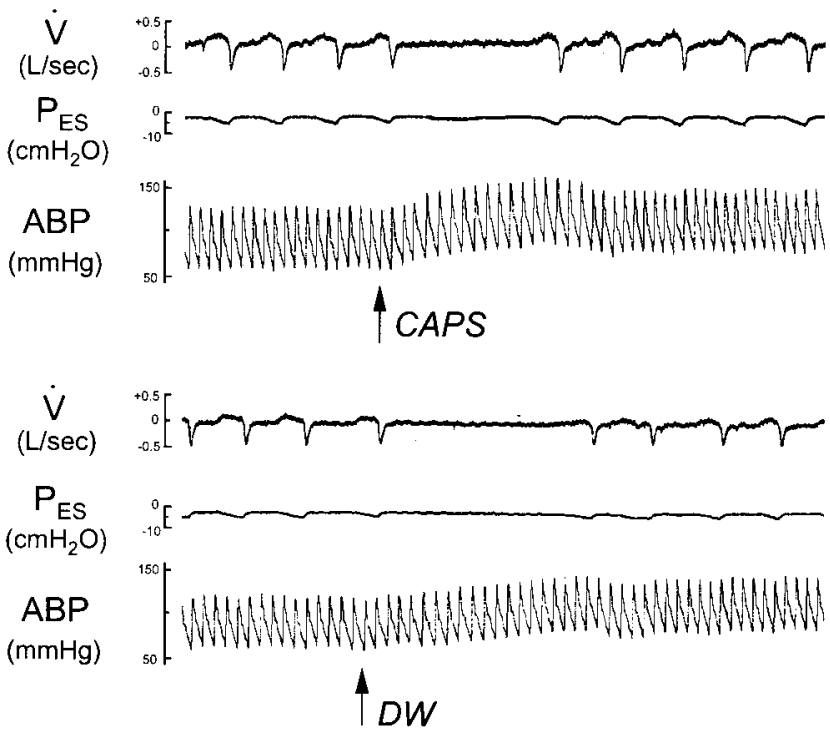

After perineural CAPS

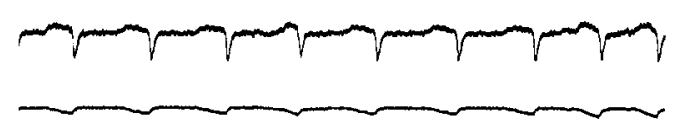

Mth

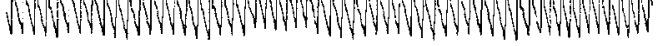
† CAPS

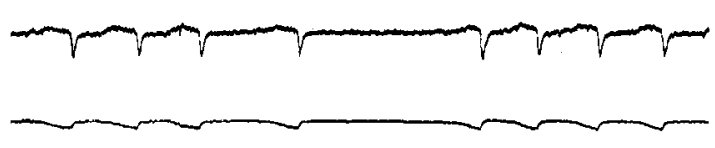

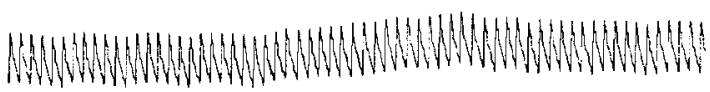

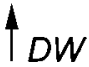

$\stackrel{5 \mathrm{sec}}{\longmapsto}$

Fig. 1. Cardiopulmonary reflexes of CAPS instilled into the larynx before (left panel) and 20 min after (right panel) perineural CAPS placement in an anesthetized spontaneously breathing dog. $\dot{\mathrm{V}}_{\mathrm{E}}=$ respiratory airflow; $\mathrm{P}_{\mathrm{ES}}=$ esophageal pressure; $\mathrm{ABP}=$ arterial blood pressure.
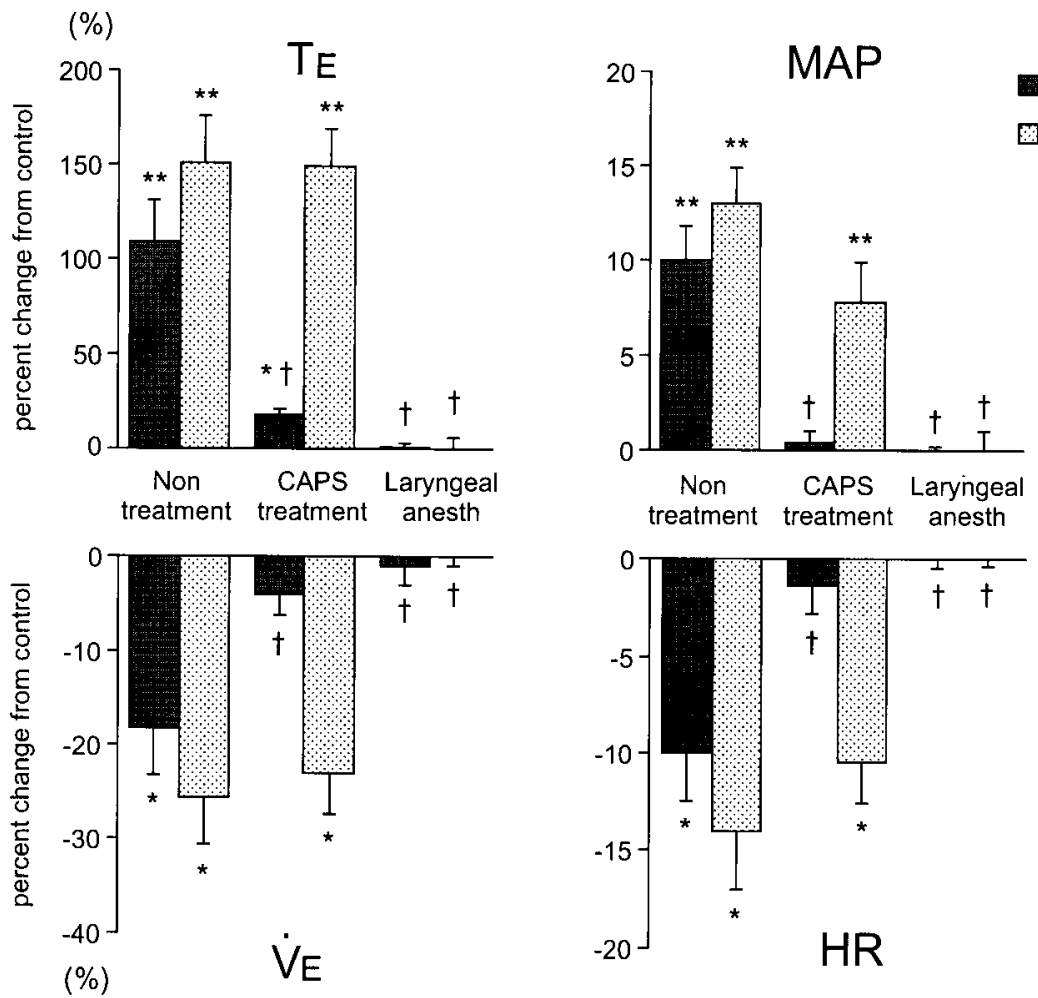

Fig. 2. Effects of perineural CAPS treatment to the SLN on peak changes in expiration time $\left(\mathrm{T}_{\mathrm{E}}\right)$, expired ventilation $\left(\dot{\mathrm{V}}_{\mathrm{E}}\right)$, heart rate (HR) and mean arterial blood pressure (MAP) elicited by CAPS and DW instilled into the larynx. Data were expressed as a percent change from control. $* * P<0.01, * P<0.05$ different from control values $\left(\mathrm{T}_{\mathrm{E}}\right.$ and $\dot{\mathrm{V}}_{\mathrm{E}}$ : consecutive breaths before instillation, MAP and HR: $60 \mathrm{sec}$ before instillation); ${ }^{\dagger} P<0.05$ different from non-treatment. 
accepted as a potent stimulant of unmyelinated CAPSsensitive C-fibers and DW is a potent stimulant of 'waterresponsive', presumably myelinated, receptors such as rapidly adapting receptors (RARs) and respirationmodulated pressure or drive receptors associated with either the lack of chloride anions or hypoosmolarity [10]. In fact, the laryngeal CAPS-sensitive receptors have little or no response to DW, and water-responsive receptors vice versa from the single unit recordings of the SLN in dogs [4].

There are several reports with regard to the selective block of C-fiber afferents by perineural CAPS treatment: Schelegle et al. [11] have demonstrated that perineural CAPS selectively blocked both the $\mathrm{C}$-wave components and C-fiber chemoreflexes evoked by CAPS $(20 \mu \mathrm{g} / \mathrm{ml})$ in dogs. Naida et al. [7] have shown that an apneic response to laryngeal CAPS instillation $(20 \mu \mathrm{g} / \mathrm{m} l)$ were blocked by perineural CAPS treatment $(100 \mu \mathrm{g} / \mathrm{m} l)$ to the SLNs in rats. In more recent report in dogs using the same experimental setting as present study, we described that the perineural CAPS treatment $(100 \mu \mathrm{g} / \mathrm{ml})$ to the SLNs considerably reduced the $\mathrm{C}$-wave afferent compound action potentials [6]. Taken together, it is presumed that the dose of perineural CAPS treatment $(100 \mu \mathrm{g} / \mathrm{m} l)$ used in this study may work on blocking C-fiber's reflexes at least up to 20 $\mu \mathrm{g} / \mathrm{m} l$. If such was the case, then it would be expected in this study that the cardiopulmonary reflexes responses to CAPS $(10 \mu \mathrm{g} / \mathrm{m} l)$ that were blocked by perineural CAPS treatment may result from stimulation of laryngeal C-fibers. A mild but significant expiratory prolongation (19\% increase of control, $P<0.05$; Fig. 2) which was still present even after the CAPS treatment may be mediated by activation of thin myelinated fibers, since CAPS applied directly onto the airway mucosa also activates a portion of $\mathrm{A}-\delta$ fibers [8]. By contrast, the reflex responses to DW instillation that were unaffected by the perineural CAPS treatment but completely blocked by laryngeal anesthesia would be due to stimulation of laryngeal water-responsive receptors.

In summary, the marked cardiopulmonary reflexes to laryngeal CAPS and DW instillations, the responses which are separated by perineural CAPS treatment to the SLNs, may result from stimulation of different sensory afferents such as CAPS-sensitive C-fibers and water-responsive receptors.

ACKNOWLEDGMENTS. The authors are grateful to Dr. Shirou Suzuki, The University of Tokyo Veterinary Medical Center, for assistance with anesthesia and surgery. T. Mutoh is supported by a Research Fellowship of the Japan Society for the Promotion of Science for Young Scientists.

\section{REFERENCES}

1. Hishida, N., Tsubone, H. and Sugano, S. 1997. J. Vet. Med. Sci. 59: 499-501.

2. Mutoh, T., Nishimura, R., Kim, H.-Y., Matsunaga, S. and Sasaki, N. 1997. Am. J. Vet. Res. 58: 885-890.

3. Mutoh, T., Tsubone, H., Nishimura, R. and Sasaki, N. 1997. J. Vet. Med. Sci. 59: 801-806.

4. Mutoh, T., Tsubone, H., Nishimura, R. and Sasaki, N. 1998. Respir. Physiol. 111: 113-125.

5. Mutoh, T., Kanamaru, A., Suzuki, H., Nishimura R, Sasaki, N. and Tsubone, H. 1999. J. Vet. Med. A 46: 335-343.

6. Mutoh, T., Kanamaru, A., Nishimura R, Sasaki, N. and Tsubone, H. 2000. J. Vet. Med. Sci. 62: 117-120

7. Naida, A. M., Ghosh, T. K. and Mathew, O. P. 1996. Respir. Physiol. 103: 11-17.

8. Riccio, M. M., Kummer, W., Biglari, B., Myers, A. C. and Undem, B. J. 1996. J. Physiol. (Lond.) 496: 521-530.

9. Richerson, G. B. and Getting, P. A. 1992. Brain Res. 591: 7987.

10. Sant'Ambrogio, G., Tsubone, H. and Sant'Ambrogio, F. B. 1995. Respir. Physiol. 102: 1-16.

11. Schelegle, E. S., Mansoor, J. K. and Green, J. F. 1995. J. 
Appl. Physiol. 79: 600-606. 